\title{
"Things Are as Numerous as Strings of Pearls, as Numerous as the Brilliant Flowers"-Arts of Figure of Speech in Qian Zhongshu's Novel Fortress Besieged
}

\author{
Jiu-gen $\mathrm{XIAO}^{1}$ and $\mathrm{Xi}$-juan $\mathrm{CHEN}^{2}$ \\ ${ }^{1}$ Jiangxi Normal University, College of Liberal Arts, Nanchang Jiangxi 330022; \\ ${ }^{2}$ Nanchang Institute of Science and Technology, Education Department for Ethnic \\ Minorities, Nanchang Jiangxi 330108
}

Keywords: Fortress besieged, Language, Arts in figure of speech.

\begin{abstract}
Fortress Besieged is a famous novel written by Zhongshu Qian. The most prominent feature in this work is its witty expressions with figure of speech used for the characterization, which is bound to do research. The paper uses comparison and induction in the research. It is concluded that the language art of the figure of speech has some characteristics: the figure of speech is used in everywhere in the novel; the figurative imagery is far from the original meaning, but suitable in the description. From the perspective of linguistics, this paper focuses on the language arts, accompanied by some figures, which is unique in the research.

Qian's Fortress Besieged is an excellent novel in the history of modern Chinese literature, which a profound analysis of irony for life, and a classic art with colorful words and expressions [1]. Its success is with multi factors, and the most important aspect is the use of language art. The expressions are wit, sharp, profound, humorous, in particular, figure of speech is amazing.

Figure of speech is regarded as the oldest, the most common used rhetorical way, and it uses the similar nature of things to describe things or explain the reasons [2]. Because of its features of fresh and natural, vivid and strange, the novel has always been loved by the people. Some people even think that a creative simile or metaphor not only has a strong visual impact, but also gives the impression that lingering aftertaste. Qian Zhongshu is fond of the figure of speech. He believes that, "figure of speech is characteristic of literary language [3]," Thus, there are many expressions in figure of speech in his novels, poetry, essays and other works. He also believes that, "many of the ideas are based on system of figure of speech [4]." Thus, the wonderful words and expressions in his works are also based on his deep understanding of figure of speech.

His novel Fortress Besieged is in the case, and the expressions in figure of speech are brilliant.

The rhetorical art of Fortress Besieged is like a blend of Chinese and Western cultures. According to rough statistics, the use number of figure of speech in Fortress Besieged is up to more than 700 , almost in every page, every paragraph, which is called the garden of figure of speech. The forms of the figure of speech are diversified, just as Liu Xie in Wenxin Diaolong from Southern Liang Dynasty put it, "The figure of speech appear in the sound, in appearance, in the heart, or in the material [5]."

Then, what kind of areas is the art of Fortress Besieged embodied in? The examples are shown as follows.
\end{abstract}

\section{Figure of Speech Used in Fang Hongjian}

Fang Hongjian is the leading man in the novel, whose character runs through the 
novel, so there are a lot of simile and metaphor expressed through his thoughts and words. He is a complex character, so it is shown dialectically in the work. His intelligence, kindness and sincere pursuit of pure love, disgusting philistine culture are promoted in the novel, but his struggling for his hesitation, cowardice and weakness, character can not get rid of the outside interference are the satire in the work. And this irony is achieved through a variety of forms in figure of speech.

For example, Fang did not pay attention to the study when he was study abroad, until he came back homeland under the dual pressures of his father and father in-law, he realized the importance of diploma. At that moment, such a figure of speech was used to describe the situation as follows:

This diploma has the function just like the function of the piece of leaf for Adam and Eve, which can cover the ugly part; the small piece of paper can also cover a person's ugly and stupid. A man without a diploma is just like a man is mentally naked without a cover.

At first glance at this analogy, people can not help laughing and then admire the author's rich imagination, because he can connect the diploma of students with Adam and Eve's leaves for covering. On reflection, it is not unreasonable. One is used to cover the shame of a body, and the other is used to cover the spiritual shame. Leaves for Adam and Eve are seldom mentioned, because most people think it is very vulgar, but the author can accurately depicts the image of a fake diploma by using the leaves used to cover the shame of a body, readers could not help laughing. The author is in pursuit of meaning likeness, rather than the pursuit of its shape likeness. As Liu Xie says, "Things in different appearance but same context inspire the thoughts of the author, and then author should take the figure of speech to make the analogy."[5] It is shown in Fig.1.

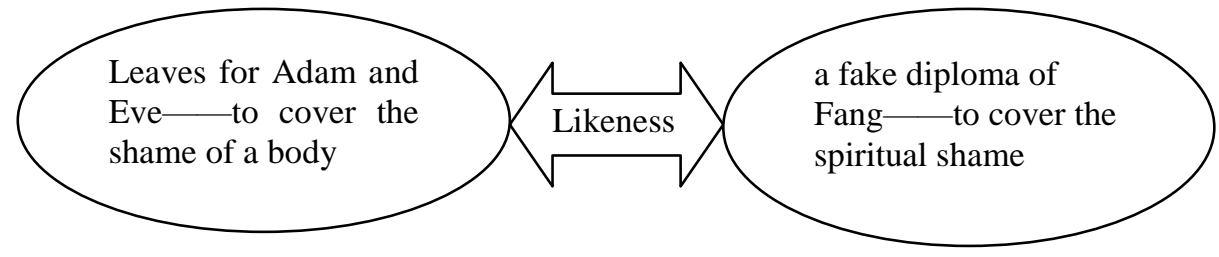

Figure 1

After this, there is another passage like this:

Fang Hongjian wonders that, it is undoubtedly the mischief of Irish people, but he bought a fake diploma from Irish people to coax people in hometown, so he is also a liar. But ---- remember, Fang had been to the Department of Philosophy ---- cheating sometimes is not immoral. Plato in Utopia mentioned, the soldiers should lie to their enemy; doctors lie to patients, officials lie to the public. Saint Confucius also pretended to be ill to coax sad Confucianism and Mencius even lie to King Qi Xuan for pretending to be ill. Father and father in-law wanted Fang to be a doctor, can he let them down? So Fang bought a fake diploma to coax them, like people in Qing Dynasty spend money to buy a official position in the government, or British colonial merchants serve the imperial treasuries to the government to buy the title of knight, which is a honor to the family, but also the duty of the son to the parents.

The writer makes an analogy between people buying officials in the history of Chinese and foreign countries with Fang buying a fake diploma, then profoundly exposes the hypocrisy of some people in the community, stubborn evil of self-deception, and those descriptions completely torn people's fake " packaging" into pieces. 
After Fang bathing, he back to the cabin, lied down and sat up, then dispelled the idea to lie. It seems the same uncomfortable as the pregnant woman who wants to do abortion, perhaps Miss Pao had no intention of that words......

This is the description of Fang's hesitant, and he wonders whether to go to Miss Pao's cabin. The writer takes the situation of dispelling the idea as the situation of a woman in pregnancy who wants to do abortion. The analogy is very vivid. Accordingly, it is not difficult to imagine the pain of Fang in his mood at the moment.

......Flicking down the pace, as if a step is riding in mind, when the pace stops in halfway, it seems the heart stops to move, not even to bear the pressure, fortunately people continues to accelerate the pace which approaches to ......

These words are written to describe Fang's psychological changes in the hallway when he heard footstep approaching to. He was hesitant to go to Miss Pao's cabin: if he goes there, he can be afraid that Miss Pao did not want him be there; does not go, he can not be reconciled, he can be afraid that he will miss the opportunity. In this contradiction occasion, footsteps were heard in the corridor, as if from Miss Pao's cabin, no wonder Fang felt as if the pace of that step were riding in his heart.

Mr. Qian is knowledgeable and familiar with many ancient classics and modern customs and culture, from which people can find all sources of the figure of speech used in his words. He is good at grasping characteristics of things and then makes an analogy. As in the novel, Su Wenwan took the initiative to kiss, then Fang kissed Su Wenwan reluctantly. At that time, the description is like this:

This kiss is very simple and light in a small spot, just as if some visitors' lips gently touch the rim of tea cups without drink in the official situation of Qing Dynasty, or the witness gently touch the "Bible" to take an oath in the western court, at best, like those believer who kiss Tibet Buddha or Pope's big toe. It is a close by keeping somebody at a distance.

The "kiss" here is used three images to make the analogy: the ancient Chinese officialdom entertainment, witnesses under oath western court, and foreign various rituals, in order to show the emotional nature of Fang to Miss $\mathrm{Su}$---- He did not love Miss Su. Although kiss is a way for men and women to enjoy pleasure, but Fang did not kiss in heart, because he was not that fond of Miss $\mathrm{Su}$, this kiss is without feeling, is a kiss with distance, as believers who kiss Tibet Buddha or Pope's big toe. This simile is very apt to show a psychological behavior of Fang, but also a satire for his weak, slick and sly behaviors. Those images are from all times in different areas of level. They are very interesting, and good for academic research. This use of the rhetorical arts make the expression vivid and interesting with unique, humor style, which shows the unique language features in the history of the modern novel. It can be shown in Fig.2.

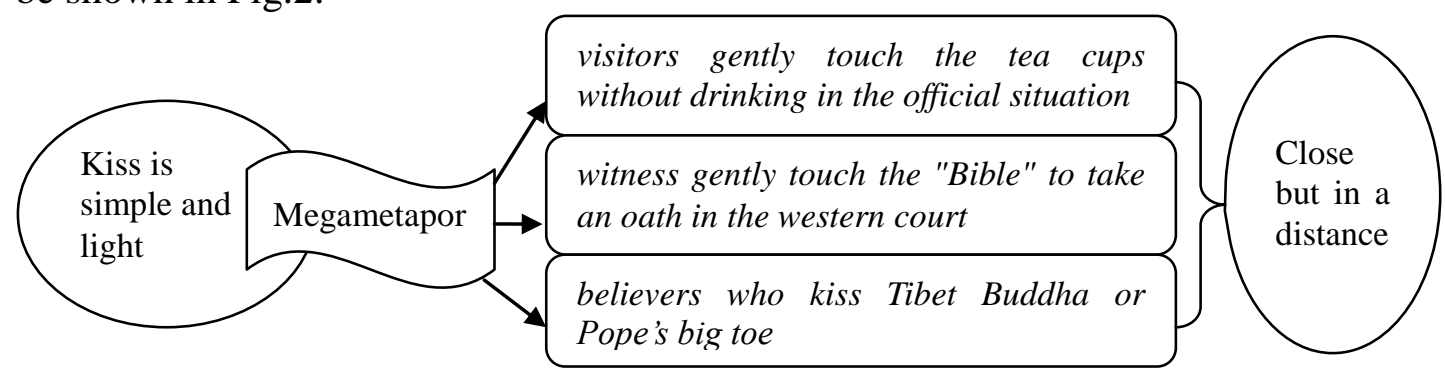

Figure 2

When Fang knows that Tang Xiao-fu does not have a boyfriend, he becomes excited, "smiling emerges from the heart to face like the bubble floating." Fang seems 
like Columbus who discovered the New World, and he can not help chuckling, after all, in that instant, there may be a reality that he can be her boyfriend, and he smiles like "bubbles" from the heart to the face.

But after Miss Tang and Fang broke up, then Miss Tang fell into the infinite pain and contradiction: "she can not forget him, and the pain is like the ache of the tooth cavity after the teeth is pulled, or like the ache of potted trees which are pulled from the pots, and pots would be broken." Here is more suitable and painful when describing Miss Tang's pain, as the tooth pain which can always remind her. Then even more profoundly: the "flower pot" is a metaphor image of Miss Tang's heart, and "trees" is Fang Hongjian; Fang had be rooted in her heart, but the heart had no place for him, so pull him away, then the heart was broken. It makes the penetrating portrait on the kind of girls with love-hate psychological romance.

In the novel, after Fang is rejected by Miss Tang, there is certain description like this:

When some one fell out of love, they would immediately show to the public like a beggar shows his rotten legs, asking for sympathy, or like the old soldier's old scar, showing for other's admiration. Fang Hongjian only hopes to hide in mental darkness, as if the sick eyes is afraid of light and the broken flesh is afraid of the wind.

This passage shows the romance modality of various broken-up couples with a series of metaphors, some like beggars, some like soldiers, but Fang hided in mental darkness, like the sick eyes is afraid of light and the broken flesh is afraid of the wind, showing his distinctive tragically sad romance mentality. The use of figure of speech is not only in the character's appearance, but deep into the soul, which let the reader feel at this moment, in the situation, what is the hero's love and hate, what is his pursuit and what does he escape from. Such mental activity description is both concise and expressive, giving a clear impression to the readers.

\section{Figure of Speech Used in Zhao Xinmei}

Zhao Xinmei is also a leading role in Fortress Besieged. This figure is an important role in the novel regarded as a second leading man, which is not the irony character in the whole novel. He gives people the overall impression with warm, generous, imposing appearance. There are many classic figure of speech used to describe him.

When he first met Fang, the description is like this:

Zhao Xinmei takes Hongjian's hand, looking at him from head to toe, like Fang is a easy carton book for kids......

How strange and interesting this analogy is! Taking Fang as a "carton book", the "book" in Zhao's eyes of course is easy to read, without any complicated words. How can a "carton book" make a comparison with famous classic work? This simile explains that Fang is pale and vulnerable in Zhao Xinmei's eyes, and the analogy obviously shows the scorn and jealousy of Zhao Xinmei in a subtle but complex way.

Then the description continues:

Zhao Xinmei is already cocky, when he heard Fang returned with Miss Su in the same boat, and his expression seems to say Hongjian is just like the light air, Zhao can not see him.

This analogy is not a conventional metaphor. Sometimes people use abstract, unreal things to compare to the concrete, tangible things, which can heighten the atmosphere of cross-sectional figures and reveal the mentality to explain the philosophy of life, and to give the readers rich imagination. From those words, people can see Fang's pale in Zhao's eyes again [6]. 
He is like a large hollow turnip with a big body but empty mind

Zhao compared him to a hollow turnip, without the slightest malice, but a little tease of meaning.

He is good at making a speech with foreign words, those American words are like loud fluent rollover of the heart, rubbed with oil, polished with wax, which can slide over half of the space.

This analogy is very consistent with the identity of the so-called politicians---sharp-tongued, thick-skinned but belly empty, even though his voice is loud, impassioned, long-winded, in full flight, but the invisible empty of his words is like the invisible sound thunder.

I regret that I could not ask people to record your voice into a tape at that moment. If that came true, that voice would be quite different, which is like sound with wind and sea wave, like sound for eating in a terrible hurry with a scream in it, fluctuated with high and low and without a break. Sometimes the scream is like a wire high up, high up, then being too thin, as thin as a kite line which is going to break off, but, then it becomes stable and comes down, just like going up to a mountain top then going down.

This passage is described by Zhao Xinmei to show Fang's snoring. A series of similes can vividly illustrate Fang's snoring. It can be shown in Fig.3.

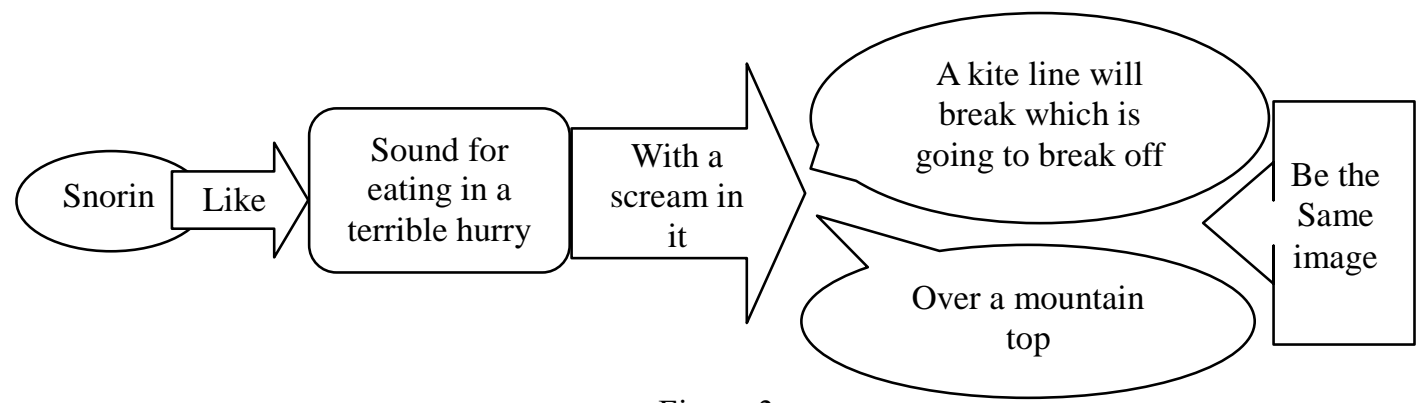

Figure 3

\section{Figure of Speech Used in Miss Pao}

Miss Pao is a character appearing at the beginning of the novel. About Miss Pao, there is a classic metaphor people always talk about:

She wears red bra, navy blue tight-fitting pants, and red toe nails revealing from the hollowing white shoes.

This western-style appears in Miss Pao in the 30s of China is very pleasing and make the current public very uncomfortable, because at that time people prefer the style of "being courteous" and "laughing without revealing the teeth". So,

Someone called her "cooked meat" because only cooked meat with warm color would be displayed on public; some people call her "truth", because it is said, "the truth is naked." Miss Pao was not naked, so people amended it into "partial truths."

"Cooked meant" is very vulgar, and very fit the image of Miss Pao. The "cooked meat with warm color" is used to describe Miss Pao's exposed arm, which is very vivid. The "cooked meat" can also cause people's appetite, and Miss Pao's half-naked can also inevitably arouse the attention of a group of people around her and arouse their desires. "Partial truth" is very elegant, which can remind people of "truth", and then "naked truth", the naked people, at last Miss Pao's exposed parts of the body. This wild imagination can not only remind the reader of the body features, but also produce a vivid humor of beauty. Thus, the figure of speech in Fortress Besieged have achieved a harmony between "vulgar" and "elegant". [7] 
When Fang and Miss Pao get together into a Western restaurant:

The soup is cold, while the ice cream is hot. The fish is like the Marines who have landed for a few days; meat like submarine soldiers who would stay long under the water; bread, butter, wine are all sour except vinegar.

The overstatement, analogy and other rhetorical skills are used here to make a satire on the poor quality food in the western restaurant.

\section{Figure of Speech Used in Sun Roujia}

Sun Roujia is the fourth woman associated with Fang in the novel. When shaping the characters, the writer did not use any direct denigration and mocking words, instead of using comparison to make a satire on her. In the novel, people regard Miss Sun as an inexperienced little girl at the first impression. As in the boat, when Fang said he fell down and almost embedded in the whale's tooth:

To her surprise, Miss Sun' eyes are round like the "O” painted by Giotto under the shining lights......

The painted "O" by Giotto is used to describe Miss Sun's eyes in surprise, exaggerated but it shows the Miss Sun in Fang eyes at the moment is a simple, naive girl, which have a contrast with the Miss Sun in the latter part of the fiction. Later, she becomes very calculating.

Miss Sun - alas! This woman is cunning enough. I am tricked ---- Miss Sun is like the whale, opening the mouth, and, you idiot are like the boat, sent to the whale's mouth.

This is the evaluation of Zhao Xinmei on Miss Sun on the ship, and she takes Sun Roujia as a whale in the sea for predation, although there is some exaggeration, it is a very accurate description of Roujia's ambitions to seize the love of Fang Hongjian.

Miss Sun thought the great nobleman refers to herself, so she put head down and a burst of red appears in the face. After hearing the news, it is not the thing that she thought, then she felt the red in her face disappears immediately, just like the warm air breathed by people to the glass.

It shows facial expressions of Sun Roujia, who is quite scheming and knowledgeable. That noticeable blush appears and disappears, which is as accurate as the barometer, reporting the inner side of her vanity. ${ }^{[6]}$

Roujia said, "How about me?" without revealing any emotions on her face, like window blinds down. But Hongjian know that this is the silence before the storm.

Using the description "window blinds down" to express Roujia's facial expressions is very appropriate.

She seemed to grab a rope with Hongjian, trying to pull each end of the rope with Fang. When the rope became straight and would be break off, she will come to Fang, and then the rope can be bent and soft. In the anger mood, they intend to quarrel, but after the quarrel, they all felt tired and empty, like the feeling after the finish of drama and sobering up after the drinking. The quarrels before going back Shanghai are simple, and they were easy to get well with each other after the quarrels, like the rich people's food, which do not stay overnight. Gradually the hatred of the quarrels need a whole day to clear up, even letting it go, and they can talk even without compromising.

In the above passage, pulling a rope and rich people's food is used to compare to the quarrel of the couple, which are humorous and strange.It is shown in Fig.4. 


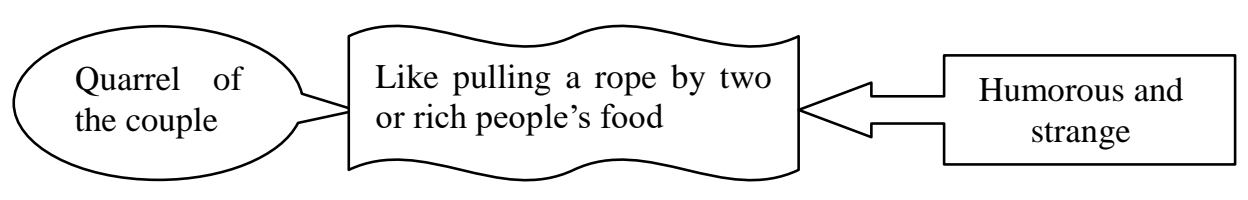

Figure 4

In the novel, the description of Sun Roujia is through making an ironic contrast of before and after. Before the engagement, she is simple, naive, cute and want Fang make a decision on everything; after the engagement, she becomes scheming and makes every attempt to tame Fang, although often quarrels with Fang, she is very good at grasping the timing. From the before and after comparison, a true representation of a flesh and blood character emerges in the novel.

\section{Conclusion}

The famous ancient Greek philosopher Aristotle said, "The figure of speech is a sign of genius." This novel Fortress Besieged vividly demonstrates the language master Qian Zhongshu's talent on the figure of speech. Its rhetorical art embodies the following characteristics: (1) the images in the figure of speech are extremely complex and diverse, and almost anything can find the metaphor in the figure of speech; (2) the relation between the noumenon and metaphor object is not that close, such as comparing going abroad to having smallpox or measles. The metaphor is strange but interesting, which can quickly and firmly arouse the reader's attention, expressing a strong aesthetic appeal.

Fortress Besieged can be regarded as a treasure of language arts, and this article only elaborates some tentative research on their artistic figure of speech; that is, just picking up a few shells on the sea shore. Other scholars can still dig the language treasure from the novel.

\section{References}

[1] Zhong-shu Qian. Fortress Besieged, People's Literature Publishing House, Beijing,2003.

[2] Bo-rong Huang, Xu-dong Liao. Modern Chinese, Higher Education Press, Beijing, 2011.

[3] Zhong-shu Qian. Seven Decorated Sets (Revised), Shanghai Ancient Books Publishing House, Shanghai, 1994.

[4] Zhong-shu Qian. Qian Zhongshu Anthology, Inner Mongolia People's Publishing House, Hohhot, 1997.

[5] Xie Liu. Wenxin Diaolong, China Social Sciences Publishing House, Beijing, 2005.

[6] Xue-chang Wang. Figure of speech in Fortress Besieged, Journal of Chengdu Teachers Training College, 3(1994).

[7] Zhi-jian Jin. On Language Features of Fortress Besieged, Masterpieces Review, 4(1994). 